\title{
Simultaneous Geometric and Radiometric Calibration of a Projector-Camera Pair
}

\author{
Marjan Shahpaski ${ }^{1}$, Luis Ricardo Sapaico ${ }^{2}$, Gaspard Chevassus ${ }^{1}$, and Sabine Süsstrunk ${ }^{1}$ \\ ${ }^{1}$ School of Computer and Communication Sciences, EPFL \\ ${ }^{2}$ Océ Print Logic Technologies S.A. \\ \{marjan.shahpaski, gaspard.chevassus, sabine.susstrunk\}@epfl.ch, ricardo.sapaico@oce.com
}

\begin{abstract}
We present a novel method that allows for simultaneous geometric and radiometric calibration of a projectorcamera pair. It is simple and does not require specialized hardware. We prewarp and align a specially designed projection pattern onto a printed pattern of different colorimetric properties. After capturing the patterns in several orientations, we perform geometric calibration by estimating the corner locations of the two patterns in different color channels. We perform radiometric calibration of the projector by using the information contained inside the projected squares. We show that our method performs on par with current approaches that all require separate geometric and radiometric calibration, while being more efficient and user friendly.
\end{abstract}

\section{Introduction}

3D sensing is gaining momentum with the increase of computational power and the possibility to display and fabricate the results with high fidelity. Structured light (SL) systems are among the most commonly used for 3D object scanning because they can be built using off-the-shelf components. A basic SL setup is made up of a single camera and a single projector that are geometrically calibrated (see Figure 11. The depth estimation principle is analogous to a dual camera stereo system, where one camera is replaced by a projector. This is possible since the geometric image formation of the projector is equivalent to that of the camera. The role of the projector is to encode the measured scene with a known pattern that can be easily decoded and identified in the camera image, allowing for dense pixel correspondences.

A thorough classification and explanation of the structured light coding techniques is presented by Salvi et al. [17]. Discrete coding methods are generally constructed through the projection of a limited set of highly contrasting

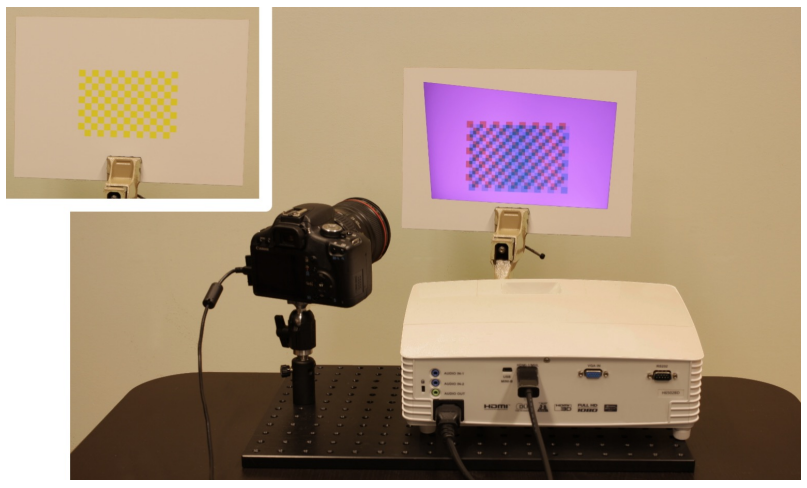

Figure 1: A setup for our simultaneous geometric and radiometric calibration of a projector-camera pair. The underlying printed pattern is shown in the inset.

intensities. If we take binary time-multiplexing codes as an example, the value that each pixel can take is either 0 or 1 , as these are the most contrasting values. On the other hand, the continuous coding methods can use the full range of intensity values (e.g., sinusoidal waves), where each pixel can take a value in the range $[0,1]$. These methods require the projection and capture of linear intensity signals, and any non-linear shift between the input values and the decoded values leads to shifted correspondences between the projected and captured images, illustrated in Figure $2 \mathrm{a}$. Therefore, in addition to geometric calibration, the continuous coding methods also require a radiometric calibration.

Radiometric calibration models the input to output intensity response of a device, because its true values are seldom known beforehand. While linear intensity images are in general easy to obtain with today's cameras, the projected images normally undergo non-linear processing imposed by the projector. The most common non-linear processing consists of applying a global tone-mapping power-law expression (also called gamma expansion) to the input intensities before projection. To linearize the projected images, the input images need to be subjected to gamma compression. 
Geometric calibration of a projector-camera pair has to be performed on a regular basis, especially while changing the parameters of the system (baseline, focal length, etc.) or if the measurement setup needs to change locations. Also, for setups that are built with lower grade materials and tools, calibration needs to be performed more often. Radiometric calibration can become invalidated through time, depending on the current temperature of the projector and age of the lamp. Therefore, we are strongly incentivized to design simple and fast calibration procedures that are easy to use even outside of research environments.

In this paper, we present a novel method for joint geometric and radiometric calibration of a projector-camera pair. Our method does not require specialized hardware, and calibration is achieved in less than one minute. We prewarp and align a specially designed projection pattern onto a printed pattern of different colorimetric properties. After capturing the patterns in several orientations, we perform geometric calibration by estimating the corner locations of the two patterns in different color channels, and we perform radiometric calibration of the projector by using the information contained inside the projected pattern squares. Our experiments show that we obtain equivalent results to the state-of-the-art methods.

\subsection{Previous Work}

Geometric Calibration The research on geometric calibration of projector-camera pairs has produced several classes of methods: two step methods [3, 9], juxtaposed methods [4, 6, 11], methods based on structured light projection [12, 15, 18, 19, 20], and methods that leverage different color channels [3, 20]. All projector-camera approaches require a printed chart that carries features with known 3D locations, and a projected chart or a projector-camera pixel correspondence map that is used for the calibration of the projector.

Two step methods rely on first calibrating the camera and then using the calibrated camera to calibrate the projector. These approaches are obviously time consuming since two sets of images have to be acquired. The juxtaposed methods position the printed and the projected patterns such that the interference of the markers of the two patterns is minimized. Correct positioning is achieved by either prewarping the projected pattern by a homography that positions it in the correct location when projected, or by projecting it right next to the printed pattern such that there is no overlap. When positioning the two patterns next to each other, a wider field of view camera lens is required, which leaves certain regions of the camera image not utilized when performing the depth estimation by SL projection. The methods that utilize prewarping require the calibration board to be relatively static for at least two consecutive image acquisitions as the prewarp is invalidated by movement.

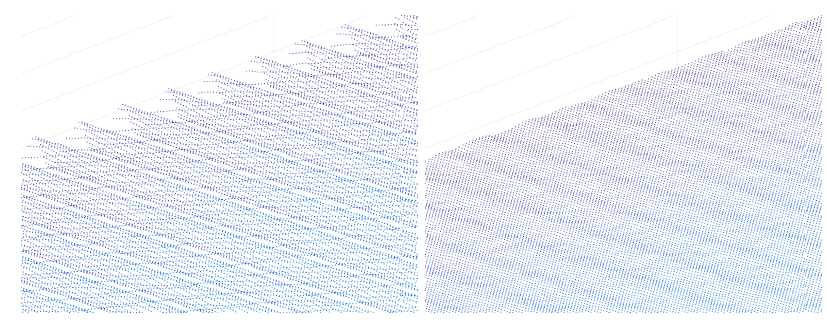

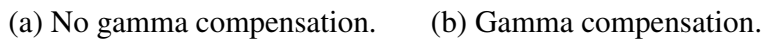

Figure 2: Systematic reconstruction errors of a flat surface induced by the non-linear processing of the input images by the projector. For this example, a conventional phaseshifting encoding was used [17].

The methods based on structured light projection tend to establish dense projector-camera correspondences, i.e., they try to relate each projector pixel to a camera pixel. The SL is projected over a printed calibration chart, whose features are later used for the calibration of both the camera and the projector. Robust SL codes require the projection of a multitude of patterns, and this process has to be repeated for each orientation of the printed calibration board. This makes them slow, data intensive, and require an immobilization of the calibration chart for the duration of the SL encoding. The methods that leverage different color channels design printed and projected calibration charts of complementary colors that minimize the crosstalk between the two charts. One obvious drawback of this class of methods is the color channel crosstalk that can lower the contrast and therefore the precision of the corner detection. In this paper, we provide a better colorimetric encoding strategy.

Radiometric Calibration Prior work on radiometric calibration mostly focuses on cameras. Debevec et al. [7] describe an approach that estimates the response curve of a camera by taking multiple images of a static scene with different amounts of exposure. Subsequent works [13, 14], propose different models for the response curve (gamma, polynomial, non-parametric, PCA based). These works are not directly applicable to a projector calibration, as projectors cannot measure scene radiance, however they do provide valuable insight into the complexity and effectiveness of different techniques. In [16], Nayar et al. perform radiometric calibration of a projector by projecting a succession of different intensity images. They later store the camera responses to those inputs in an inverse lookup table. We adopt a similar approach, however, we estimate a gamma function from the simultaneous display of different intensities.

Given the above classification of methods for geometric calibration, our approach can be classified as a juxtaposed method that leverages different color channels. This fusion, together with an innovative projection pattern design (discussed in section 33, facilitates the geometric calibration and allows for simultaneous radiometric calibration. 


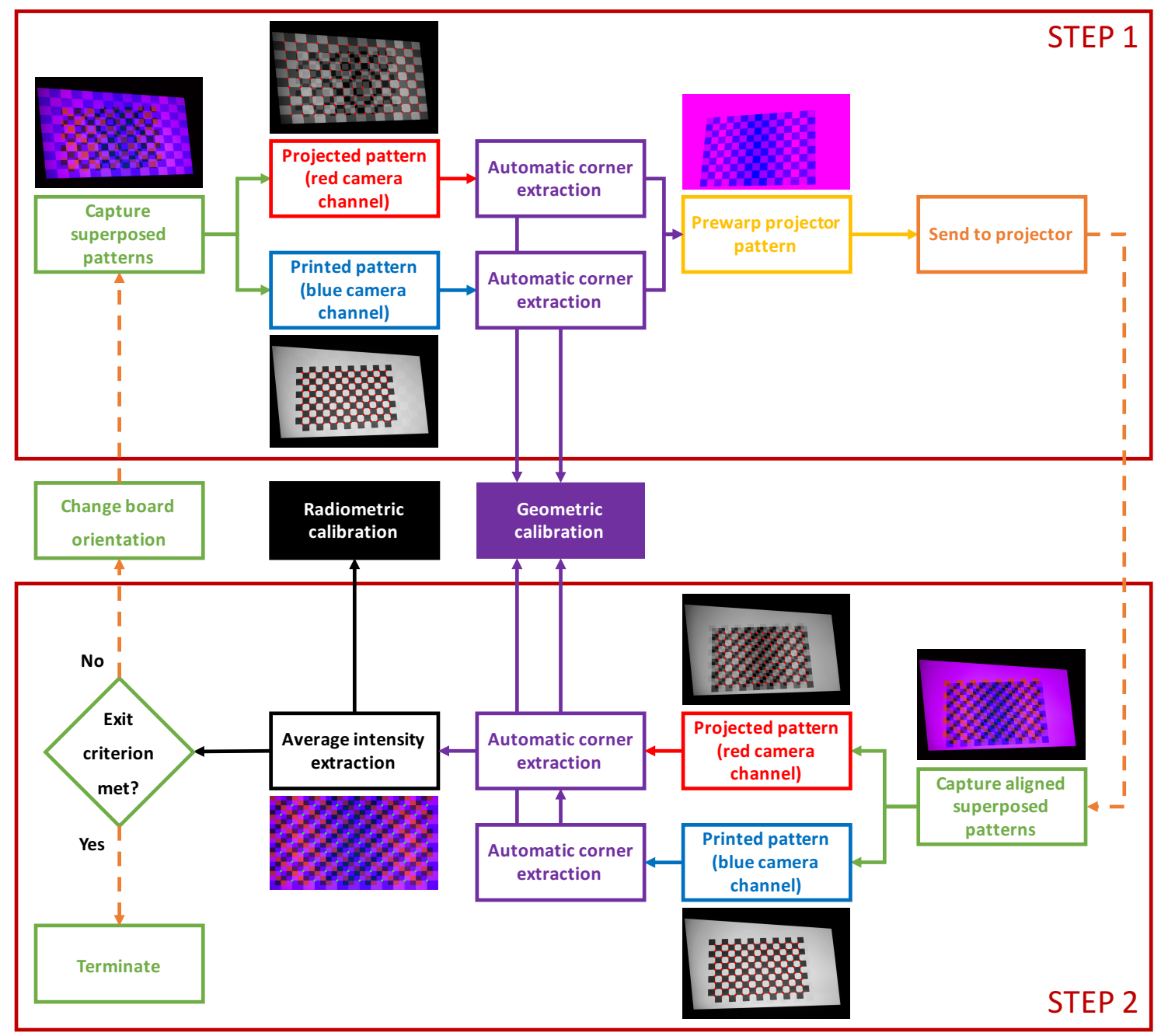

Figure 3: Flow chart of our proposed method for simultaneous geometric and radiometric calibration of a projector-camera pair. Each orientation of the calibration board requires two images. In step 1 we start by capturing an image of the arbitrarily aligned printed and projected patterns. We detect the corners of the two patterns and compute a prewarp that aligns them. In step 2, we capture an image of the aligned patterns. This allows us to extract projected intensities for radiometric calibration in addition to the corner information used for geometric calibration.

\section{Algorithm Overview}

An overview of our algorithm is provided in Figure 3 For each orientation of the calibration board we consecutively execute steps 1 and 2 . We start by projecting a specially designed checkerboard pattern, described in detail in Section 3 , on top of a conventional checkerboard pattern that is printed with a complementary color to the projected one. In step 1, we capture an image of the arbitrarily aligned patterns and detect their corners. This allows us to compute a composition homography that relates the projector image to the printed pattern. We use this homography to compute a prewarp of the projected pattern, such that when projected it lands right on top of the printed pattern, with a shift of half a printed square. Prewarp has already been employed by Audet et al. [4] and Chen et al. [6] for camera-projector geometric calibration, however, none of those methods perform radiometric calibration.

At step 2, we capture an image of the aligned patterns. After the printed and projected patterns are aligned and shifted by half a square, we extract the information encoded in the projected squares that land on white (empty) squares of the printed pattern. This information encodes the projector response to a range of different input values, which we then use for radiometric calibration.

The above process is repeated for different calibration board orientations until the desired number of loops has been acquired, usually between 10 and 15 . 


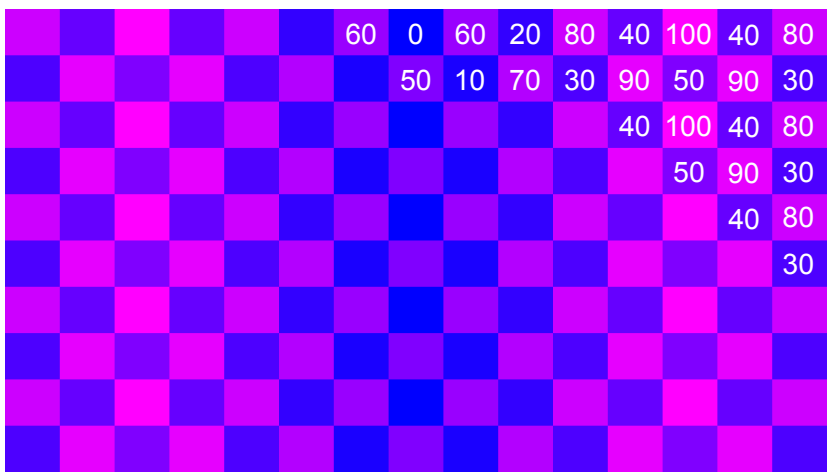

Figure 4: The projected pattern used for geometric and radiometric calibration. The red pixel intensities (in \%) of the squares vary across the chart, which is leveraged for the radiometric calibration of the projector. The values in the top-right corner are example intensities in the red channel. The blue pixel intensities are constant.

\section{Design of Calibration Patterns}

Printed Pattern The printed pattern is a standard checkerboard pattern comprised of white and yellow squares. Its configuration is equivalent to that of the projected pattern (Figure 4p, but it features only fulltone squares. An example printed pattern can be seen in the inset of Figure 1 We opted to use yellow squares because the yellow colorant is almost transparent to the red and green wavelengths, but it absorbs in the blue wavelengths. Therefore, the yellow squares show as dark squares in the blue channel of the camera, where we can easily detect the checkerboard pattern (see Figure [5b). While [3] have used cyan and white squares, we opted for a yellow-white configuration, because the real yellow inks are in general much closer to the perfect yellow ink than cyan inks are. Yellow ink is much more transparent in the green-red wavelength range (500$700 \mathrm{~nm}$ ) than the cyan is in the blue-green range (400-600 $\mathrm{nm}$ ). This translates into a fairly visible ghost of the cyan pattern in the blue image channel - the channel where the projected pattern should be isolated.

Projected Pattern The projected pattern is shown in Figure 4. It is specifically designed such that we can simultaneously perform both geometric and radiometric calibration of the projector. Note that the squares are colored with a bluemagenta palette. More precisely, all squares are composed of $100 \%$ blue and varying intensities of red. The top-right corner of Figure 4 shows sample intensity values for the red color channel. The patches that have higher red intensity become increasingly magenta due to the additive mix with blue. The pattern is symmetric across the middle column, and the intensity values alternate across rows.

These colors are chosen for two reasons. First, the high intensity blue is present to illuminate the printed pattern. Since the yellow squares absorb in the blue region, this increases the contrast of the printed pattern and makes the calibration independent of external illumination. Second, the squares include a red component so that we can detect the projected checkerboard corners in the red channel of the captured images where the printed pattern is rather transparent (Figure 5c). Although the squares have varying intensities of red, with our design we preserve the contrast ratio of each corner; we impose an intensity difference of at least $40 \%$ between the dark and the bright squares at a corner.

The varying intensities of red across the projected squares are used for the radiometric calibration of the projector. In the current design we have 11 intensity gradations, ranging from $0 \%$ to $100 \%$, in $10 \%$ steps.

\section{Algorithm}

Geometric calibration establishes a relationship between a set of known 3D object coordinates (from the real-world) and their corresponding set of $2 \mathrm{D}$ projections on the imaging sensor (pixel coordinates).

For camera calibration, a simple approach to establish the $2 \mathrm{D}$ to $3 \mathrm{D}$ correspondences is by capturing a printed calibration board composed of checkers, similar to the one shown in Figure 4. Since the calibration board squares have known physical dimensions, their corners provide us with the 3D object coordinates. After we detect the same corners in the captured images, i.e., we identify their 2D image coordinates, we can calibrate the camera with the objective of establishing a link between the two sets of coordinates. More formally:

$$
s \mathbf{x}^{I}=\mathbf{K}\left[\mathbf{R}^{I} \mid \mathbf{t}^{I}\right] \mathbf{X}
$$

where $\mathbf{X}$ and $\mathbf{x}^{I}$ are the 4D and 3D homogeneous coordinate vectors of the corners on the printed board, and their images in the captured image $I$, respectively. Rotation matrix $\mathbf{R}^{I}$ and translation vector $\mathbf{t}^{I}$ relate the optical center of the camera to the board origin (usually an extreme corner on the board). The $\mathbf{K}$ matrix stores the intrinsic parameters of the camera, and $s$ is an arbitrary scaling factor.

Since the board is flat, all corners of the printed pattern have equal values for the height. If we take these values to be 0 , then the relationship between the points on the calibration board and the image sensor is given by a homography:

$$
\mathbf{H}^{I}=\mathbf{K}\left[\mathbf{r}_{1}^{I} \mathbf{r}_{2}^{I} \mathbf{t}^{I}\right]
$$

where $\mathbf{r}_{1}^{I}$ and $\mathbf{r}_{2}^{I}$ are the 1-st and the 2-nd columns of $\mathbf{R}^{I}$.

The model given in Eq. (1) is linear and does not take into account the possible radial and tangential distortions imposed by the camera lens. However, they need also be 


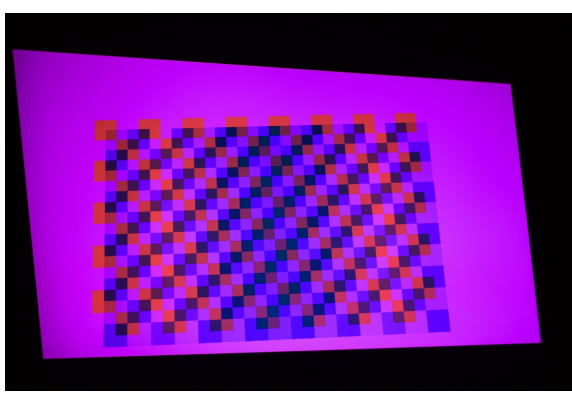

(a) Image of the superposed patterns.

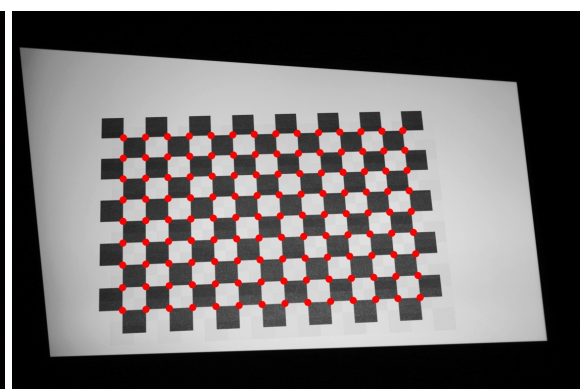

(b) Blue image channel (printed pattern).

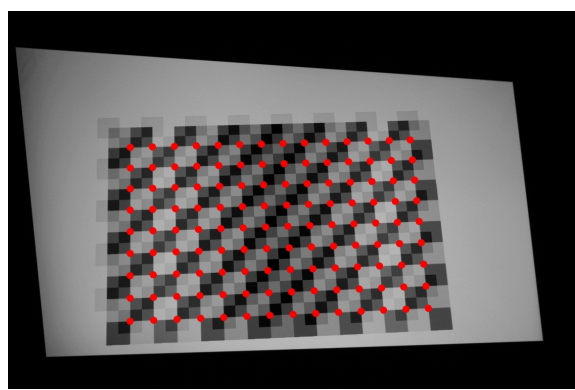

(c) Red image channel (projected pattern).

Figure 5: Captured image of an aligned superposition of the printed and the projected pattern. This is the input to step 2 of the algorithm. Corner detection for the two patterns is performed in different color channels.

estimated for an accurate camera calibration. For more details about camera calibration with flat boards, see [21].

The geometric calibration of the projector is slightly more complicated, as it cannot capture 3D coordinates from the real-world. In order to calibrate a projector, we use a calibrated camera, which captures the corners of a projected calibration pattern. Since the camera is precalibrated, we can use it to back-project rays through the captured projected corners into the $3 \mathrm{D}$ world. For accurate projector calibration it is important to account for the non-linear distortions of the camera lens when back-projecting.

To solve for the scale ambiguity of the back-projected rays, in addition to the projected calibration pattern, we also need a printed calibration pattern of known dimensions that rests on the same plane where we observe the projected pattern. Since the square dimensions of the printed pattern are known, we can calculate the rotation and translation between the board's origin and the camera optical center, i.e., we can identify the board plane in space. The calibration board is defined by its normal, given by $\mathbf{r}_{3}^{I}$, which can be computed from the known $\mathbf{K}$ and $\mathbf{H}^{I}[21]$, and a point on that plane, which is the vector $\mathbf{t}^{I}$. By intersecting the projected rays with the plane, we obtain the 3D coordinates of the projected corners. Acquiring the 2D pixel coordinates for the projector is straightforward, since we already have a noiseless version of the calibration pattern that we project.

Radiometric calibration establishes an input to output intensity response of a display or a capture device. Off-theshelf display devices (monitors and projectors) rarely have the option of projecting linear images, thus we observe a non-linear output response. The most commonly observed response is a power function, also called a gamma function. This is mostly due to conformity with the sRGB standard [2], whose overall gamma is approximately $\gamma=2.2$. However, we do not radiometrically calibrate the camera [7, 13, 14], as most consumer cameras can capture linear images $(\gamma=1)$. We focus solely on estimating the gamma of the projector.
The algorithm that we describe below is fully automatic except when the operator has to physically change the orientation of the board that carries the printed calibration pattern. We have made the code for the calibration procedure publicly available 1

\subsection{Image Capture}

For programatically capturing images we use the gPhoto2 toolbox [1], as it provides complete control over the camera settings. It is also very general and supports almost all models from major camera manufacturers.

\subsection{Corner Detection}

Given the colorimetric properties of our printed and projected patterns (described in Section 3), we use the blue channel of the captured images to extract the printed corners. Figure $5 b$ shows the blue channel for the image in Figure 5a As expected, the printed pattern is completely isolated from the projected pattern. These favorable conditions allow us to use an automatic checkerboard detector [8]. The detected corners are marked with red dots on Figure $5 b$.

Figure $5 \mathrm{c}$ shows the red channel of the image in Figure $5 \mathrm{a}$. As expected, the corner contrast suffers from the specific design of the projected pattern, especially in the darker regions. This is mainly due to the gamma of the projector which compresses the dark tones, and extends the bright. Also, a faint ghost of the printed pattern can be seen, since the yellow ink is slightly absorbing in the red wavelengths.

Analogous to the printed corners, for detecting the projected corners in step 1 of the algorithm we also utilize automatic checkerboard detector. In step 2, since the prewarp forces the projected pattern corners to land between the corners of the printed pattern, we implemented a search around the expected locations of the projected corners. We use the Harris corner detector [10] to find the projected corners with subpixel accuracy.

\footnotetext{
${ }^{1}$ http://ivrl.epfl.ch/research/grc
} 


\subsection{Prewarp}

Having extracted the corners of the printed and the projected patterns, we can compute homographies that relate both the camera to the physical printed/projection board, $\mathbf{H}_{c b}$, and the camera to the projector image, $\mathbf{H}_{c p}$. Building a composition of these homographies provides us with a homography that relates the printed pattern to its current location on the projector image:

$$
\mathbf{H}_{b p}=\mathbf{H}_{c b}^{-1} \cdot \mathbf{H}_{c p}=\mathbf{H}_{b c} \cdot \mathbf{H}_{c p}
$$

We use this homography to compute a prewarp of the projected image such that when projected, it lands right on top of the printed pattern with a shift of half a printed square. We perform the shifting by subtracting the distance of half a printed square from the 3D positions of the board corners when estimating $\mathbf{H}_{c b}$.

\subsection{Geometric Calibration}

To perform the actual geometric calibration we use Bouguet's camera calibration toolbox [5], which implements Zhang's [21] algorithm. In addition to the intrinsic parameters, the algorithm also provides us with the extrinsic parameters that link the camera position and orientation to that of the calibration board, for each calibration board orientation. The minimal number of board orientations is 2 (for a simplified camera model with rectangular pixels). For more general models with radial and tangential distortions, 10 or more orientations are recommended, also due to noise in the corner detection phase.

Once we have the camera calibrated, we project rays through the pixel coordinates of the corners of the projected images and intersect them with the corresponding plane that carries the printed pattern to compute their 3D positions in the camera coordinate frame. By using the corresponding rigid transforms we then translate those coordinates into the board coordinate frame and calibrate the projector using the same procedure we used for the camera.

\subsection{Radiometric Calibration}

As discussed in section 3 each square of the projected pattern is encoded with a red value that varies across squares. To easily extract the intensity value of each square in the captured image, we undo the prewarp by fitting the image of the projected pattern onto the original pattern, i.e., we apply the inverse of $\mathbf{H}_{c p}$. Figure 6 shows an example rectified projected pattern.

Of interest to us is the subset of corners that emerge by the intersection of the projected corners, specifically on top of the white squares of the printed pattern. These corners are characterized by having grid indexes of different parity. Thus, we denote this set of corners as $\kappa$, and denote a particular corner as $\kappa_{i j}$.

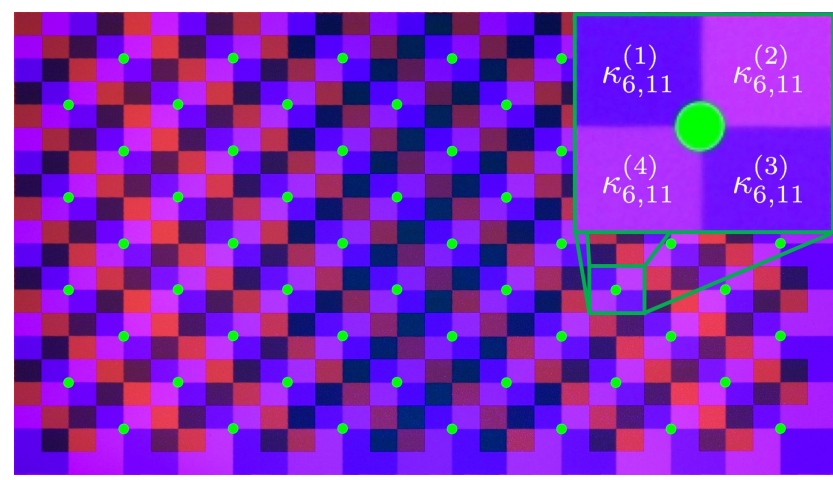

Figure 6: The projected pattern in its original size, after having been warped into position atop the printed pattern. The green markers, $\kappa_{i j}$, indicate the corners of the projected pattern that overlap with white squares on the printed one. The inset shows the values associated with an example corner $(i, j)=(6,11)$.

Each $\kappa_{i j}$ has four adjacent squares. We denote the topleft square as $\kappa_{i j}^{(1)}$ and continue clock-wise, i.e. the bottomleft is $\kappa_{i j}^{(4)}$.

Each square has two values associated with it, the projected red-channel intensity and the captured one. Let us denote the projected square intensity as $P_{i j}^{(k)}$.

Due to light variation and other sources of noise, the pixel intensity of a square in the captured image is not uniform, and we therefore consider its mean. We denote the mean intensity value of square $\kappa_{i j}^{(k)}$ in the captured image as $C_{i j}^{(k)}$.

Given $P$ and $C$ we estimate the response function of the projector by fitting a power function onto the data:

$$
\underset{a, b, \gamma}{\operatorname{argmin}} \sum_{k=1}^{4}\left\|C^{(k)}-a \cdot\left[P^{(k)}\right]^{\gamma}-b\right\|^{2}
$$

We perform this non-linear least squares optimization for each image $I$ in the calibration set, and thus obtain a $\gamma$-value per image, which we denote $\gamma^{I}$.

Finally, we compute the expected gamma value for all images:

$$
\gamma^{\star}=\mathbb{E}\left[\gamma^{I}\right]
$$

Note that our method focuses on extracting the data, which allows for any other non-linearity to be used for explaining the response of the projector. For that purpose, Eq. 4 needs to be updated to reflect the new model. 


\section{Experiments}

We implemented our method in Matlab. During the experiments, we captured linear RAW images, which we demosaiced in order to extract the different color channels. In the current implementation of the algorithm, the calibration is performed at the end after the desired set of orientations has been acquired. However, this can easily be modified in order to have a running update of the calibration parameters and the reprojection error, since the corner detection runs while capturing the images.

On the hardware side, we used an AcerH6502BD projector (color, DLP, 1920 x 1080 px) and a Canon T1i digital camera (color, CMOS, 4752 x 3168 px) with a Canon EF 24-105mm f/4L IS USM lens. The physical calibration board was arranged in a configuration of $15 \times 10$ squares, where each square is $9 \mathrm{~mm}$ in size. Throughout the testing the calibration board was kept at a distance of approximately $45 \mathrm{~cm}$ from the camera and the projector. An overview of the calibration setup can be seen in Figure 1 .

We capture 2 images per calibration board orientation, which is on par with most other methods (see Table 1). However, we perform geometric and radiometric calibration at the same time, whereas the other methods only perform geometric calibration. For these methods, adding radiometric calibration means that another calibration procedure needs to be performed. Our method is much less time consuming then capturing the two calibration image sets separately, which requires change of the calibration board and possibly camera exposure parameters.

Geometric calibration For the geometric calibration of the camera, we achieve an average reprojection error of $0.25 \mathrm{px}$ and a very low standard deviation of 0.03 px over multiple calibrations. The calibration of the projector was slightly less accurate, with an average reprojection error of $0.46 \mathrm{px}$ and a standard deviation of $0.08 \mathrm{px}$. This is an expected result, since the accuracy of the projector calibration depends on the accuracy of the camera calibration. In addition, due to the brightness of the projector, we set the camera aperture

Table 1: Reprojection errors (RMSE in pixels) for camera and projector calibration and the number of images per orientation as reported by the authors.

\begin{tabular}{cccc}
\hline Authors & Camera & Projector & imgs./or. \\
\hline M. Ashdown [3] & 0.25 & 0.47 & - \\
S. Audet [4] & 0.33 & 0.20 & 2 \\
C.-Y. Chen [6] & - & 0.188 & 2 \\
A. Griesser [9] & $<0.4$ & $<1.5$ & multiple \\
Z. Li [12] & 0.09 & 0.149 & 12 \\
D. Moreno [15] & 0.329 & 0.145 & $\approx 40$ \\
Ours & $\mathbf{0 . 2 5}$ & $\mathbf{0 . 4 6}$ & $\mathbf{2}$ \\
\hline
\end{tabular}

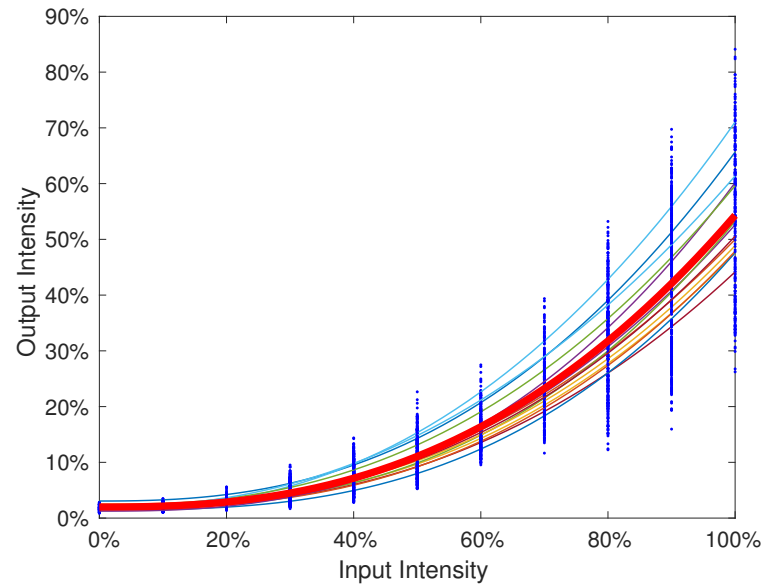

Figure 7: Gamma estimation for 14 image calibration set. The thick red line is the mean gamma curve. The thin lines represent the estimated gamma curves for each individual image. The vertically distributed blue dots show the input vs. output pixel intensity values over all images.

to $f / 22$, whereas the projector had an aperture of $f / 2.56$. The large aperture of the projector translates into shallow depth of field, which deteriorates the corner detection accuracy.

Table 1 shows our results in comparison with the stateof-the-art calibration results reported in the respective publications. Only the best calibration results are shown, even if several were reported. It can be seen that our results are on par with the current methods for geometric calibration.

Radiometric calibration As a ground truth for the radiometric calibration, we projected 11 consecutive grayscale images with input intensities ranging from $0 \%$ to $100 \%$, in $10 \%$ steps, over a uniformly white screen. We then captured the projections with our camera, and fitted a gamma function to the captured data. This approach is similar to [16]. We denote this method as the ground truth method. We estimated the gamma on different locations across the projection screen. The computed mean gamma is 2.57 , with a standard deviation of just 0.03 . The low standard deviation indicates that the projector gamma is fairly uniform across the screen.

We then performed radiometric calibration with our proposed method by capturing 14 images at different orientations. These are the images from step 2 of our algorithm, which are also used for geometric calibration. Figure 7 graphically summarizes our results. Each blue vertical bar represents the extracted output intensities across all captured images for a single input intensity. The thick red curve is the average power function, denoted by $\gamma^{\star}$ in Eq. 5. The 14 thin curves are the gamma functions estimated for each image individually. The mean gamma is 2.53 , with a standard deviation of 0.15 . This result is in accordance with the gamma we computed with the ground truth method. 


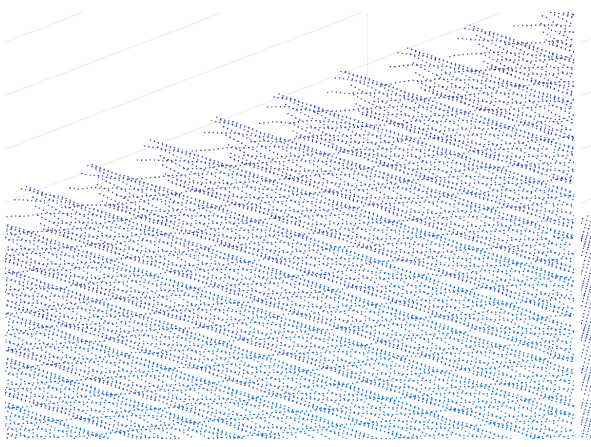

(a) No gamma compensation.

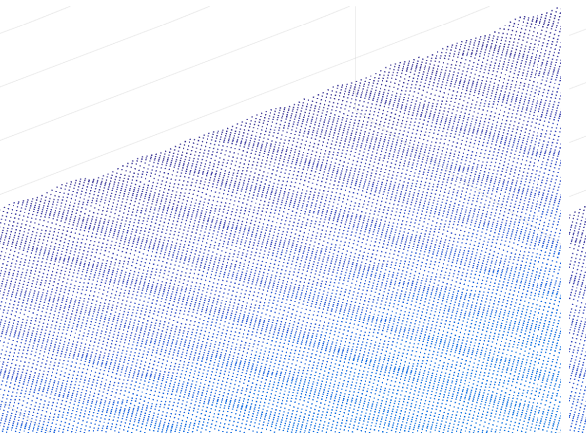

(b) Ground truth method.

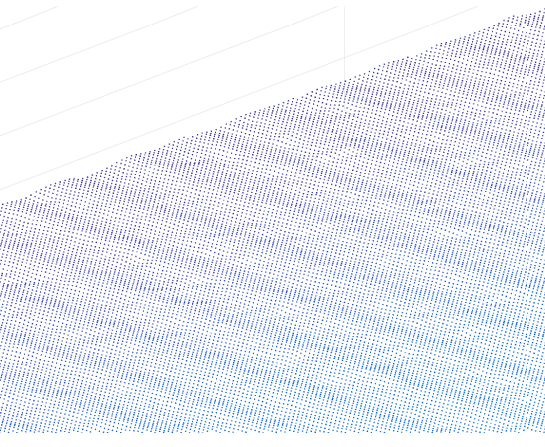

(c) Our method.

Figure 8: Systematic errors in the reconstruction of a flat surface. (a) Non-gamma compensated projection patterns exhibit large depth variations $(\mathrm{RMSE}=0.331 \mathrm{~mm})$. (b) Gamma compensated patterns, gamma computed with the ground truth method $(\mathrm{RMSE}=0.056 \mathrm{~mm})$. (c) Gamma computed with our proposed method $(\mathrm{RMSE}=0.055 \mathrm{~mm})$.

The spread of the fitted gamma curves in Figure 7 is mainly due to changes in the scaling and bias parameters of the fitted functions (parameters $a$ and $b$ in Eq. 4). This happens because the captured intensities are scaled by the different orientations that the projector and the camera take with respect to the calibration board (changes in irradiance). Gamma has no effect on the extreme (0\% and $100 \%)$ input intensities, but only on the intermediate values. Furthermore, the coefficient of variation of gamma across the images is $6.1 \%$, whereas for the scaling parameter it is $15.1 \%$.

Planarity test We performed a planarity test to validate the performance of the radiometric calibration in a real-world scenario. The test consists of fitting a plane into a point cloud and estimating their fit. Therefore, we reconstructed a flat board by using a phase-shifting SL code. This is a continuous coding method and is based on the projection of sinusiodal patterns (see detailed explanation in [17]).

We performed three reconstructions of the flat surface, of size $10 \times 10 \mathrm{~cm}$, by projecting: linear patterns, gamma compensated patterns with gamma computed using the ground truth method, and gamma computed using our proposed method. The reconstructed point cloud for each of the methods can be seen in Figure 8. As expected, the non-gamma compensated patterns exhibit the largest depth variations, produced by erroneous correspondences between the linear input and the non-linear output patterns. The gamma compensated patterns, on the other hand, show a much lower degree of variation.

Finally, we computed the RMSE of fitting a plane into the point clouds. The error for the linear patterns amounted to: $0.331 \mathrm{~mm}$; for gamma estimated with the ground truth method: $0.056 \mathrm{~mm}$; and gamma estimated with our proposed method: $0.055 \mathrm{~mm}$. The 6 fold decrease in reconstruction error strongly reinforces the need for radiometric calibration, i.e., gamma estimation and compensation.

\section{Limitations}

The presented method also has some limitations. First, we assume that the gamma is constant across the surface of the projector and across different color channels, which might not be true for lower quality equipment. In addition, severe vignetting of the camera and of the projector can also decrease the accuracy of the radiometric calibration. Initial full illumination images can therefore be taken to compensate for vignetting before projecting the patterns. Excessively specular calibration boards can degrade the quality of the extracted radiometric values. The guided detection of the projected corners in step 2 will not work well for projectors with strong radial distortions.

\section{Conclusion}

In this work we present a novel method that allows for geometric calibration of a projector-camera pair, simultaneous with a radiometric calibration of the projector. The method is automatic, simple, and efficient compared to other calibration methods, since it does not have an inherent overhead of projections and captures. It is faster compared to dedicated geometric and radiometric calibration techniques as it requires less manual manipulation together with a decreased complexity of a single image capturing and processing cycle. System recalibration can thus be performed more often in order to guarantee consistent results over time. We have also shown that the method offers high quality calibration results and performs on par with the current projector-camera calibration methods.

In addition, we demonstrated that radiometric calibration is essential for achieving high depth estimation accuracy with SL systems. Due to their numerous advantages, the continuous coding techniques represent the most popular choice for scene encoding, which makes our method very attractive to the $3 \mathrm{D}$ reconstruction community. 


\section{References}

[1] gphoto2 toolbox.

[2] M. Anderson, R. Motta, S. Chandrasekar, and M. Stokes. Proposal for a standard default color space for the internet - sRGB. In IS\&T Color and Imaging Conference (CIC'96), pages 238-245, 1996.

[3] M. Ashdown and Y. Sato. Steerable projector calibration. In IEEE Computer Vision and Pattern Recognition Workshop (CVPRW'05), pages 98-98, 2005.

[4] S. Audet and M. Okutomi. A user-friendly method to geometrically calibrate projector-camera systems. In IEEE Computer Vision and Pattern Recognition Workshop (CVPRW'09), pages 47-54, 2009.

[5] J.-Y. Bouguet. Camera calibration toolbox for matlab. 2004.

[6] C.-Y. Chen and H.-J. Chien. An incremental target-adapted strategy for active geometric calibration of projector-camera systems. Sensors, 13(2):2664-2681, 2013.

[7] P. E. Debevec and J. Malik. Recovering high dynamic range radiance maps from photographs. In ACM International Conference on Computer Graphics and Interactive Techniques (SIGGRAPH'97), pages 369-378, 1997.

[8] A. Geiger, F. Moosmann, Ö. Car, and B. Schuster. Automatic camera and range sensor calibration using a single shot. In IEEE International Conference on Robotics and Automation (ICRA'12), pages 3936-3943, 2012.

[9] A. Griesser and L. Van Gool. Automatic interactive calibration of multi-projector-camera systems. In IEEE Computer Vision and Pattern Recognition Workshop (CVPRW'06), pages 8-8, 2006.

[10] C. Harris and M. Stephens. A combined corner and edge detector. In Citeseer Alvey Vision Conference (AVC'88), volume 15, page 50, 1988.

[11] T. Hurtos, G. Falcao, and J. Massich. Plane-based calibration of a projector camera system. VIBOT Master, pages 1-12, 2008.

[12] Z. Li, Y. Shi, C. Wang, and Y. Wang. Accurate calibration method for a structured light system. Optical Engineering, 47(5):053604-053604, 2008.

[13] H. Lin, S. J. Kim, S. Süsstrunk, and M. S. Brown. Revisiting radiometric calibration for color computer vision. In IEEE International Conference on Computer Vision (ICCV'11), pages 129-136, 2011.

[14] T. Mitsunaga and S. K. Nayar. Radiometric self calibration. In IEEE Computer Vision and Pattern Recognition (CVPR'99), volume 1, 1999.

[15] D. Moreno and G. Taubin. Simple, accurate, and robust projector-camera calibration. In IEEE $3 D$ Imaging, Modeling, Processing, Visualization \& Transmission (3DIMPVT'12), pages 464-471, 2012.

[16] S. K. Nayar, H. Peri, M. D. Grossberg, and P. N. Belhumeur. A projection system with radiometric compensation for screen imperfections. In IEEE International Conference on Computer Vision Workshop (ICCV PROCAMS'03), volume 3, 2003.

[17] J. Salvi, S. Fernandez, T. Pribanic, and X. Llado. A state of the art in structured light patterns for surface profilometry. Pattern Recognition, 43(8):2666-2680, 2010.
[18] J. Wilm, O. V. Olesen, and R. Larsen. Accurate and simple calibration of DLP projector systems. In SPIE MOEMSMEMS, pages 897909-897909, 2014.

[19] S. Yamazaki, M. Mochimaru, and T. Kanade. Simultaneous self-calibration of a projector and a camera using structured light. In IEEE Computer Vision and Pattern Recognition Workshop (CVPRW'11), pages 60-67, 2011.

[20] S. Zhang and P. S. Huang. Novel method for structured light system calibration. Optical Engineering, 45(8):083601083601, 2006.

[21] Z. Zhang. A flexible new technique for camera calibration. IEEE Transactions on Pattern Analysis and Machine Intelligence (TPAMI), 22(11):1330-1334, 2000. 